\title{
DIMENSIONAL CROSSOVERS IN MAGNETORESISTANCE OF SUBMICRON FILMS AND WIRES OF CdTe:In*
}

J. Jaroszyński, J. Wróbel, M. SAWicki, T. SKoŚKieWicz, G. Karczewski, T. Wojtowicz, J. Kossut, T. Dietl

Institute of Physics, Polish Academy of Sciences

Al. Lotników 32/46, 02-668 Warszawa, Poland

E. Kamińska, E. Papis, A. Piotrowska

Institute of Electron Technology, Al. Lotników 32/46, 02-668 Warszawa, Poland

R. Duś AND R. NowakowskI

Institute of Physical Chemistry, Polish Academy of Sciences

Kasprzaka 44/52, 01-224 Warszawa, Poland

We present millikelvin studies of magnetoresistance for epitaxial films and wires of $\mathrm{CdTe}: \mathrm{In}$. In comparison to the data with theoretical predictions for the weakly localized regime we put into the evidence the presence of the temperature-induced dimensional crossovers in the studied systems. Our measurements probe the electron phase-breaking rate and indicate that the main dephasing mechanism arises from electron scattering from thermal fluctuations of three- or two-dimensional electron liquid.

PACS numbers: $71.55 . \mathrm{Jv}$

Doped semiconductors have proved their usefulness for studies of the quantum corrections to the conductivity of disordered systems. It is now well established that these corrections are dominated by phenomenon known as weak localization (WL) [1], and by disorder-modified electron-electron interactions (EEI). WL arises from constructive interference of self-crossing electron paths. Such a path has its time-reversal analog with a fixed relative phase (zero in the absence of the magnetic field $B=0$ ). The resulting constructive interference gives rise to backscattering and decreases conductivity. WL is extremely sensitive to inelastic scattering events such as electron-electron and electron-phonon scattering which randomize electron phases.

*This work is supported by the Committee for Scientific Research grant No. 8-T11B-02108. 
The WL criterion for the dimensionality is that a system becomes two-dimensional if its thickness $d$ becomes smaller than $L_{\varphi}$. In the same way, a system is one-dimensional if both $d, W<L_{\varphi}$, where $W$ is sample width and $L_{\varphi}=\sqrt{D \tau_{\varphi}}$ - the phase coherence length. Here $D$ is the diffusion constant and $\tau_{\varphi}-$ the dephasing time, a mean time corresponding to a change of electron phase by $\pi$. Thus WL effects are stronger in wires and thin films than in bulk samples as the reduced space dimensionality enhances backscattering.

The length scale requirements for observing reduced dimensionality with respect to EEI are much stringent and are characterized by the thermal diffusion length $L_{T}=\sqrt{\hbar D / k T}$.

In the magnetic field time-reversal symmetry of the two paths is broken. The corresponding phase difference is $2 S / L_{m}^{2}$, where $S$ is an area enclosed by electron path and $L_{m}=\sqrt{\hbar / e B}$. The paths with area $>L_{m}^{2}$ accumulate phase greater than $\pi$ and their contribution to WL averages out leading to negative magnetoresistance. At the same time the definition of dimensionality in a magnetic field should be modified. In particular, the behavior of WL is $1 \mathrm{D}$ if $d, W<L_{\varphi}, L_{m}$ and 2D when $d<L_{\varphi}<L_{m}$. Otherwise we deal with a 3D system.

The conductivity corrections $\Delta \sigma(B)=\sigma(B, T)-\sigma(0, T)$ in a perpendicular magnetic field for 1D [2] and 2D [3] are given by

$$
\begin{aligned}
\sigma(B) & =\frac{e^{2}}{\pi \hbar}\left[L_{\varphi}-\frac{3}{2}\left(L_{1}^{-2}+\frac{W^{2}}{3 L_{m}^{2}}\right)^{-1 / 2}+\frac{1}{2}\left(L_{\varphi}^{-2}+\frac{W^{2}}{3 L_{m}^{2}}\right)^{-1 / 2}\right], \\
\sigma(B) & =\frac{e^{2}}{2 \pi^{2} \hbar} \\
\times & \left\{\psi\left(1 / 2+\frac{L_{m}^{2}}{4 L_{1}^{2}}\right)+\frac{1}{2}\left[\psi\left(1 / 2+\frac{L_{m}^{2}}{4 L_{\varphi}^{2}}\right)-\psi\left(1 / 2+\frac{L_{m}^{2}}{4 L_{3}^{2}}\right)\right]\right\},
\end{aligned}
$$

where $L_{1}^{-2}=L_{\varphi}^{-2}+L_{\text {sox }}^{-2}+L_{\text {soy }}^{-2}+2 L_{\text {soz }}^{-2}, L_{3}^{-2}=L_{\varphi}^{-2}+2 L_{\text {sox }}^{-2}+2 L_{\text {soy }}^{-2}$. Here $L_{\mathrm{sox}}=L_{\mathrm{soy}}=L_{\mathrm{so} z}=L_{\mathrm{so}}=\sqrt{D \tau_{\mathrm{so}}}$ describe the length scale for spin-orbit scattering and $\psi$ is the Digamma function.

In order to verify Eqs. (1)-(2), four-terminal transverse magnetoresistance measurements were performed in a dilution refrigerator from $T=4.2 \mathrm{~K}$ down to $0.06 \mathrm{~K}$.

The studied MBE grown [4] films are $4 \mu \mathrm{m}, 0.5 \mu \mathrm{m}$ and $0.1 \mu \mathrm{m}$ thick and have electron concentration $n \approx 10^{18} \mathrm{~cm}^{-3}$. Wires are fabricated by means of $30 \mathrm{keV}$ e-beam lithography [5], followed by wet etching in $0.5 \%$ solution of $\mathrm{Br}_{2}$ in ethylene glycol. Precise atomic force microscope (AFM) measurements showed their cross-section of side $0.3 \mu \mathrm{m}$.

As we reported previously [6], in submicron wires of CdTe:In universal conductance fluctuations (UCFs) are observed. In this paper we focus on WL magnetoresistance, which is obscured by UCFs. Thus, in order to average out UCFs, device with ten wires connected in parallel was fabricated.

Figure 1 presents corrections to the conductivity in studied films and the device formed by ten wires. Solid lines are calculated within 2D or 1D WL theory with $L_{\varphi}, L_{\text {so }}$ and $W$ as adjustable parameters. For comparison, $\Delta \sigma$ calculated from 3D model [7] is also shown. 

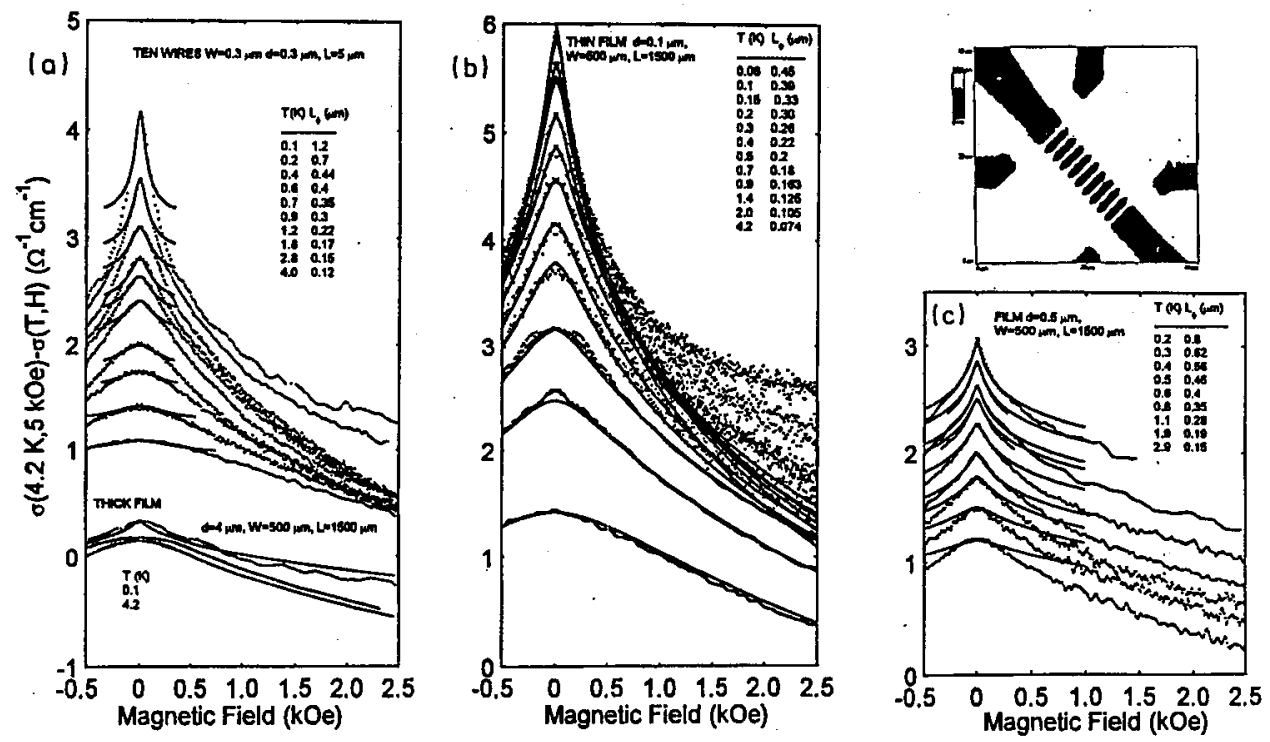

Fig. 1. Negative corrections to the conductivity as a function of the magnetic field for ten CdTe:In wires connected in parallel (a), $d=0.1 \mu \mathrm{m}$ (b), $0.5 \mu \mathrm{m}$ (c) and $4 \mu \mathrm{m}$ (a) films. Dots depict experimental points. Solid lines show magnetoconductance calculated according to the $1 \mathrm{D}(\mathrm{a}), 2 \mathrm{D}(\mathrm{b}, \mathrm{c})$ and $3 \mathrm{D}$ [bottom part of $(\mathrm{a})$ ] weak localization theory. The inset shows AFM image of the sample.

Several remarks are appropriate. All samples are well described by 3D theory at $4.2 \mathrm{~K}$ for the whole range of the magnetic field. For the thickest sample this is true down to $50 \mathrm{mK}$ so that this sample can be treated as reference 3D system.

For the remaining samples the discrepancy between measured resistance and $3 \mathrm{D}$ model increases below $\approx 3 \mathrm{~K}$. This indicates temperature crossover from 3D behavior to $2 \mathrm{D}$ or $1 \mathrm{D}$, respectively. It is evident that both the magnitude and the functional form of the magnetoresistance of 1D, 2D and bulk samples are different.

In stronger magnetic field, when $L_{m}$ becomes smaller than the thickness (width) of measured samples, the magnetoresistance is again well described by $3 \mathrm{D}$ model thus the crossover back to 3D system occurs.

Figure 2 shows inelastic scattering rates inferred from appropriate fits to Eqs. (1) and (2). For the sample with $d=0.5 \mu \mathrm{m}$ as well as for the wires we find $\tau_{\varphi}^{-1}=1.4 \times 10^{10}(T /[\mathrm{K}])^{1.45} \mathrm{~s}^{-1}$ down to $100 \mathrm{mK}$. Similar behavior was deduced from UCF correlation field temperature dependence [6]. Such a $T^{3 / 2}$ dependence agrees with theoretical prediction for electron-electron scattering process, occurring in $3 \mathrm{D}$ electron liquid, with small energy [8] transfers $(\Delta E \ll k T)$. Such a process can be treated as the Coulomb scattering of a given electron from thermal fluctuations of the electron liquid. Hence, although the samples have reduced dimensionality with respect to localization effects, they are still 3D regarding to EEI. Indeed, down to $100 \mathrm{mK}$ thermal diffusion length, $L_{T}=0.1 \times T^{-1 / 2} \mu \mathrm{m} \mathrm{K}^{-1 / 2}$, is longer than all linear dimensions of these samples. 


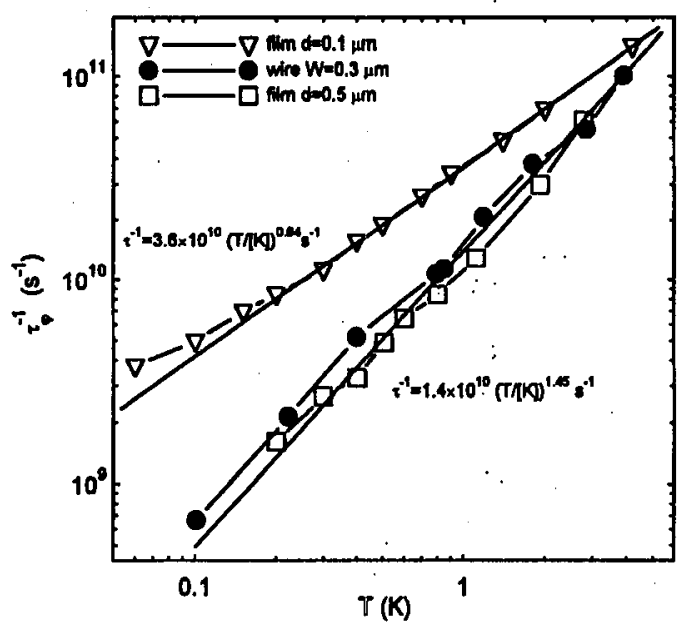

Fig. 2. Phase breaking rates as a function of temperature. Solid lines represent fits to the formula $\tau^{-1}=a T^{b}$.

The situation is different for $2 \mathrm{D}$ sample with $d=0.1 \mu \mathrm{m}$. In the latter $\tau_{\varphi}^{-1}=3.6 \times 10^{10}(T /[\mathrm{K}])^{0.94} \mathrm{~s}^{-1}$. The $\tau_{\mathrm{ee}}^{-1} \propto T$ behavior is expected for thermal fluctuations of $2 \mathrm{D}$ electron gas. $L_{T}$ becomes larger than the sample thickness below $1 \mathrm{~K}$, confirming this crossover. Similar magnitude and temperature dependence of $\tau_{\mathrm{ee}}^{-1}(T)$ was observed in GaAs/AlGaAs heterostructures [9] and thin metallic films [10].

Spin-orbit dephasing mechanism is relatively weak in our samples. Characteristic positive magnetoresistance was not observed down to $60 \mathrm{mK}$. A scattering rates for SO were found to be $\tau_{\text {so }}^{-1} \approx 5 \times 10^{8} \mathrm{~s}^{-1}$ for all studied samples. Such a value can be explained by the mechanism [11] which assumes the randomization of the spin polarization by elastic scattering due to spin-split resulting from the electric field of the $\mathrm{Cd}-\mathrm{Te}$ dipoles.

In conclusion, weak localization in low-dimensional samples was used to identification and quantitative description of electron dephasing mechanisms. Two of them play role in our samples: thermal fluctuations of interacting electron liquid and spin-orbit coupling.

\section{References}

[1] P.W. Anderson, E. Abrahams, T.V. Ramakrishnan, Phys. Rev. Lett. 43, 718 (1979); L.P. Gorkov, A.I. Larkin, D.E. Khmelnitskii, Pisma Zh. Eksp. Teor. Fiz. 30, 248 (1979) [JETP Lett. 30, 228 (1979)]; G. Bergmann, Phys. Rep. 107, 1 (1984); for review, see C.V.J. Beenakker, H. Van Houten, Solid State Phys. 44, 1 (1991).

[2] B.L. Altshuler, A.G. Aronov, Pisma Zh. Eksp. Teor. Fiz. 33, 515 (1981) [JETP Lett. 33, 499 (1981)].

[3] S. Hikami, A.I. Larkin, Y. Nagaoka, Prog. Theor. Phys. 63, 77 (1980).

[4] G. Karczewski, A.K. Zakrzewski, L. Dobaczewski, W. Dobrowolski, E. Grodzicka, J. Jaroszyński, T. Wojtowicz, J. Kossut, Thin Solid Films 267, 79 (1995). 
[5] J. Wróbel, Acta Phys. Pol. A 90, (1996).

[6] J. Jaroszynnski, J. Wróbel, M. Sawicki, E. Kamińska, T. Skośkiewicz, G. Karczewski, T. Wojtowicz, A. Piotrowska, J. Kossut, T. Dietl, Phys. Rev. Lett. 75, 3170 (1996).

[7] M. Sawicki, T. Dietl, J. Kossut, J. Igalson, T. Wojtowicz, W. Plesiewicz, Phys. Rev. Lett. 56, 508 (1986).

[8] B.L. Altshuler, A.G. Aronov, D.E. Khmelnitskii, J. Phys. C 15, 7367 (1982).

[9] K.K. Choi, D.C. Tsui, K. Alavi, Phys. Rev. B 36, 7751 (1987).

[10] M.J. Uren, R.A. Davies, M. Kaveh, M. Pepper, J. Phys. C 14, 7232 (1981).

[11] B.L. Altshuler, A.G. Aronov, D.E. Khmelnitskii, A.I. Larkin, in: Quantum Theory of Solids, Ed. I.M. Lifshits, MIR Publishers, Moscow 1982, p. 137. 